\title{
Detection of the Human Organic Anion Transporters SLC21A6 (OATP2) and SLC21A8 (0ATP8) in Liver and Hepatocellular Carcinoma
}

\author{
Yunhai Cui, Jörg König, Anne T. Nies, Marion Pfannschmidt, Michaela Hergt, \\ Werner W. Franke, Wibke Alt, Roland Moll, and Dietrich Keppler
}

Deutsches Krebsforschungszentrum, Division of Tumor Biochemistry (YC, JK, ATN, MP, DK) and Division of Cell
Biology (MH, WWF), Heidelberg, and Philipps-Universität, Institute of Pathology (WA, RM), Marburg, Germany

SUMMARY: Transport proteins mediating the selective uptake of organic anions into human hepatocytes include the organic anion transporters SLC21A6 (also termed OATP2, OATP-C, or LST-1) and SLC21A8 (OATP8). Both transporters are localized to the basolateral membrane of human hepatocytes. Because of the importance of these transporters for hepatobiliary elimination, including the removal of bilirubin and its conjugates from the blood circulation, we have generated monoclonal antibodies for studies on the expression and localization of these transport proteins. We describe two antibodies, designated monoclonal antibody MDQ (mMDQ) and monoclonal antibody ESL (mESL), directed against the amino terminus and the carboxyl terminus of human SLC21A6, respectively. Both antibodies have been characterized by immunoblot analysis, immunoprecipitation, and immunofluorescence microscopy. While mESL reacted specifically with SLC21A6, mMDQ detects both SLC21A6 and SLC21A8. Neither of the two antibodies reacted with other human, or with dog, rat, or mouse liver SLC21A family members. Antibody mMDQ may be used for the simultaneous detection of SLC21A6 and SLC21A8 in immunoblotting because of its immunoreactivity with both molecules and because of the different molecular masses of both glycosylated proteins in human hepatocytes. This is exemplified in hepatocellular carcinomas where SLC21A6 and SLC21A8 were differentially synthesized and showed an irregular staining pattern. Both transport proteins have not been detected in human hepatoma HepG2 cells. In routine paraffin sections, 10 of 12 hepatocellular carcinomas were focally positive with antibody mMDQ. In contrast, cholangiocarcinomas and liver metastases of colorectal and pancreatic adenocarcinoma were negative without exception. This suggests the usefulness of SLC21A6/SLC21A8 within a panel of tumor markers for hepatocellular carcinomas. Moreover, both antibodies should be useful in studies on the expression and localization of two important uptake transporters of human hepatocytes under physiologic and pathophysiologic conditions. (Lab Invest 2003, 83:527-538).

$T$ he removal of toxic endogenous metabolites and xenobiotic substances from the blood circulation and their secretion into bile are major functions of the liver. During this detoxification process, the uptake across the basolateral (sinusoidal) membrane and the export across the apical (canalicular) membrane of the hepatocyte play a decisive role. Whereas the export at the apical side is mediated by members of the ATPbinding cassette superfamily (Jansen, 2000; Keppler and Arias, 1997), the uptake at the basolateral side of the hepatocyte is carried out by different types of

\section{DOI: 10.1097/01.LAB.0000065015.02412.48}

Received October 7, 2002.

This article is dedicated to Harald zur Hausen on the occasion of his retirement as head of the German Cancer Research Center (Deutsches Krebsforschungszentrum) in Heidelberg with gratitude and appreciation for 20 years of leadership. This work was supported in part by Deutsches Krebsforschungszentrum, by the Deutsche Forschungsgemeinschaft through SFB 352, by the Tumorzentrum Heidelberg/Mannheim, and by the Fonds der Chemischen Industrie.

Address reprint requests to: Dr. Dietrich Keppler, Division of Tumor Biochemistry, Deutsches Krebsforschungszentrum, Im Neuenheimer Feld 280, D-69120 Heidelberg, Germany.E-mail:d.keppler@dkfz-heidelberg.de transport proteins (Kullak-Ublick et al, 2000; Suzuki and Sugiyama, 2000).

The sodium-independent uptake of many cholephilic organic anions into the hepatocyte is mediated by members of the organic anion transporter (OATP) family (Kullak-Ublick et al, 2000), classified as subfamily SLC21A within the solute carrier gene superfamily. A total of seven SLC21A members have been cloned from human tissues (Abe et al, 1999; Hsiang et al, 1999; König et al, 2000a, 2000b; Kullak-Ublick et al, 2001; Tamai et al, 2000), three of them have been established to be localized to the basolateral membrane of hepatocytes: SLC21A6 (König et al, 2000a), SLC21A8 (König et al, 2000b), and SLC21A9 (KullakUblick et al, 2001). Whereas SLC21A9 (OATP-B) has been found in a number of other tissues (Kullak-Ublick et al, 2001), expression of SLC21A6 and SLC21A8 genes is restricted to human hepatocytes, and the corresponding proteins are localized to the basolateral membrane (König et al, 2000a, 2000b). Among the human organic anion transporters identified so far, SLC21A6 is the only one shown to mediate highaffinity uptake of bilirubin and its glucuronosides in the presence of serum albumin (Cui et al, 2001b). In contrast to bilirubin, the prototypic substrate sulfobro- 
mophthalein (BSP), which has been widely used for studies on hepatic transport function (Scharschmidt et al, 1975), is a good substrate for several human SLC21A family members (Cui et al, 2001b; König et al, 2000a, 2000b; Kullak-Ublick et al, 2001), but SLC21A6 exhibits the highest affinity for BSP with a $\mathrm{K}_{\mathrm{m}}$ value of $140 \mathrm{nmol} / \mathrm{L}$ (Cui et al, 2001b). In addition to BSP, the members of the SLC21A gene family expressed in hepatocytes share a broad overlapping substrate spectrum, including $17 \beta$-glucuronosyl estradiol, estrone 3-sulfate, dehydroepiandrosterone sulfate (DHEAS), and thyroid hormones, suggesting an important function of these transporters in the detoxification of endogenous and xenobiotic substances (Cui et al, 2001b; Hsiang et al, 1999; Tamai et al, 2000).

So far, the synthesis and cellular localization of organic anion transporters of the SLC21A family in the liver has been studied with antisera generated in the rabbit (Cui et al, 2001a, 2001b; König et al, 2000a, 2000b; Kullak-Ublick et al, 2001). Because of their important role in hepatobiliary elimination and detoxification by the liver, it is of clinical interest to study the presence and the localization of these transport proteins under physiologic as well as pathophysiologic conditions. For this purpose, antisera have the disadvantage of limited availability and, in many cases, insufficient specificity. Therefore, we have generated two monoclonal mouse antibodies, directed against the amino terminus and the carboxyl terminus of SLC21A6, and characterized them by immunoblot analysis, immunoprecipitation, and immunofluorescence microscopy. Examples for the application of these antibodies are provided by studies in human liver tissue, primary hepatocellular carcinomas (HCCs), cholangiocarcinomas, liver metastases, and in the hepatoma cell lines HepG2 and PLC.

\section{Results}

\section{The Reactivity of the Monoclonal Antibodies mESL and $M M D Q$}

Two epitope-bearing peptides of human SLC21A6 were chosen for the immunization of the mice (Fig. 1): The amino-terminal peptide MDQNQHLNKTAEAQPSENKKTRYC and the carboxyl-terminal peptide ESLNKNKHFVPSAGADSETHC (König et al, 2000a). After fusion of mouse spleen cells with myeloma cells, the hybridoma cell supernatants were screened for their reactivity with human SLC21A6, both in immunoblot analyses and by immunofluorescence microscopy. After three rounds of subcloning, the clones with the highest reactivity for SLC21A6 were designated

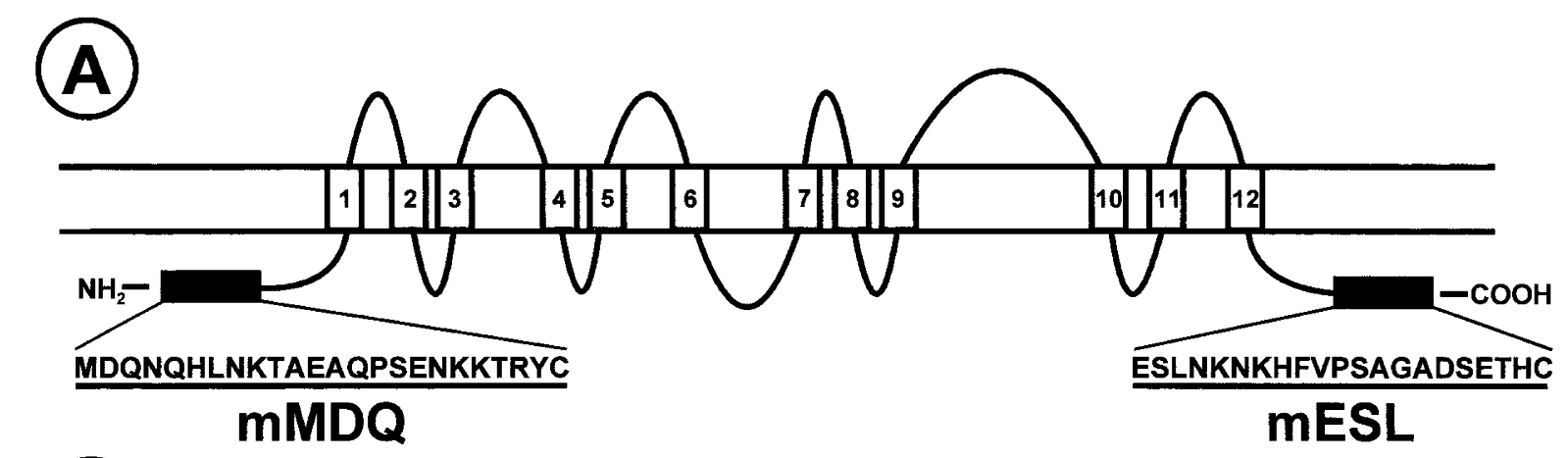

(B)

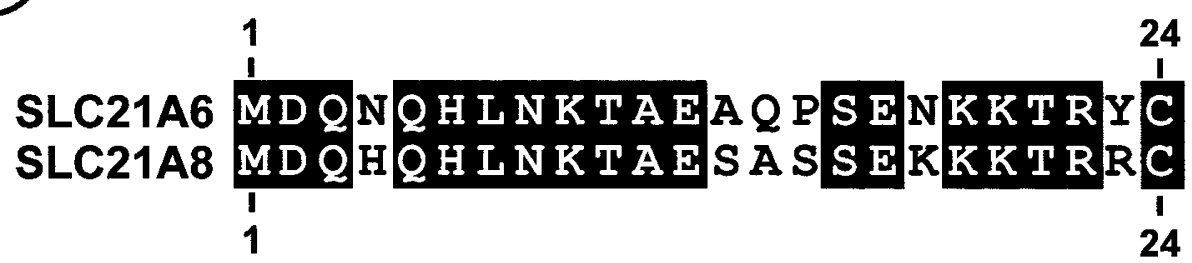

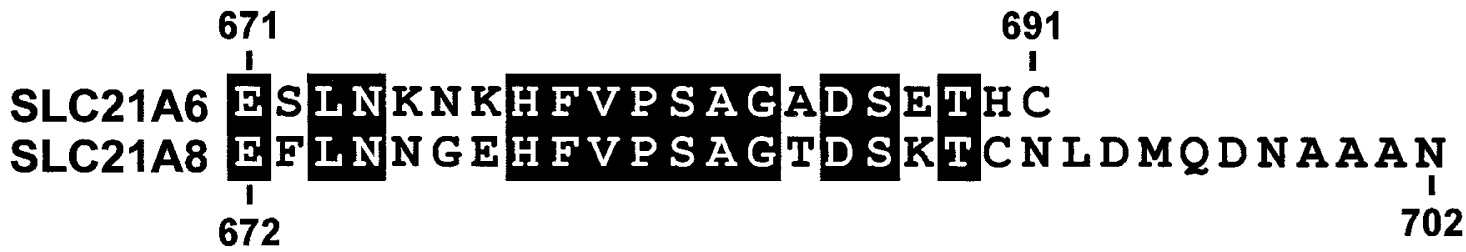

Figure 1.

Peptide sequences used for the generation of monoclonal antibodies. A, Schematic membrane topology model of human SLC21A6 (0ATP2) predicted by the TMHMM program of the Center for Biological Sequences (http://www.cbs.dtu.dk/services/TMHMM-1.0). Both peptide sequences used for the generation of the antibodies monoclonal antibody MDQ (mMDQ) and monoclonal antibody ESL (mESL) are highlighted at the amino terminus and at the carboxyl terminus, respectively. B Alignment of both peptide sequences with the corresponding peptide sequences of human SLC21A8 (OATP8). Amino acids identical in both sequences are boxed. 
monoclonal antibody ESL (mESL) (against the carboxyl terminus of SLC21A6) and monoclonal antibody MDQ (mMDQ) (against the amino terminus of SLC21A6). Antibody mESL was determined to be of IgM type and $\mathrm{mMDQ}$ of IgG type (Table 1).

Because of the high similarity between the amino acid sequences of SLC21A6 and SLC21A8, especially at the amino terminus (Fig. 1B), we examined whether the monoclonal antibodies also react with SLC21A8. As shown in Figure 2A, the mAb mESL detected SLC21A6 in the basolateral membrane (BLM) of human liver as a signal with an apparent molecular mass of about $90 \mathrm{kd}$; the additional signal detected in liver BLM with a molecular mass of about $60 \mathrm{kd}$ represents the core-glycosylated form of SLC21A6 (König et al, 2000a). In the SLC21A6-transfected HEK293 (human embryonic kidney) cells (HEK-SLC21A6), the molecular mass of the recombinant SLC21A6 is slightly higher than in the liver (Fig. 2A) as a result of different glycosylation (König et al, 2000a). No signal was detected in HEK293 cells expressing recombinant SLC21A8 (HEK-SLC21A8) by mESL, demonstrating the specific reactivity of mESL for human SLC21A6. In contrast to $\mathrm{mESL}$, the $\mathrm{mAb} \mathrm{mMDQ}$ directed to the amino terminus of SLC21A6 detected two signals in liver BLM with apparent molecular masses of about 90 $\mathrm{kd}$ and $120 \mathrm{kd}$ (Fig. 2B). Because human SLC21A8 has been shown to have a molecular mass of about 120 kd (König et al, 2000b), the 120 kd signal detected in liver BLM by $\mathrm{MMDQ}$ was likely to represent SLC21A8. This was confirmed by the reactivity of mMDQ with SLC21A8-transfected HEK293 cells. Two signals were detected in these cells with the antibody mMDQ with molecular masses of $90 \mathrm{kd}$ and $120 \mathrm{kd}$ (Fig. 2B), both of which resulted from the recombinant glycoprotein SLC21A8. The $90 \mathrm{kd}$ signal found in HEK-SLC21A8 cells corresponded to the coreglycosylated SLC21A8 (Cui et al, 2001a; König et al, $2000 b)$. Deglycosylation of the glycoproteins prepared from human liver and from transfected cells with the enzyme N-glycosidase F (Roche Molecular Biochemicals, Mannheim, Germany) resulted in a single signal detected by MMDQ with a molecular mass of about 60 kd (data not shown), similar to the molecular mass of unglycosylated SLC21A6 and SLC21A8 as calculated from their amino acid sequences.

Possible cross-reactivity of mESL with the carboxyl terminus of SLC21A9, which is also expressed in liver,

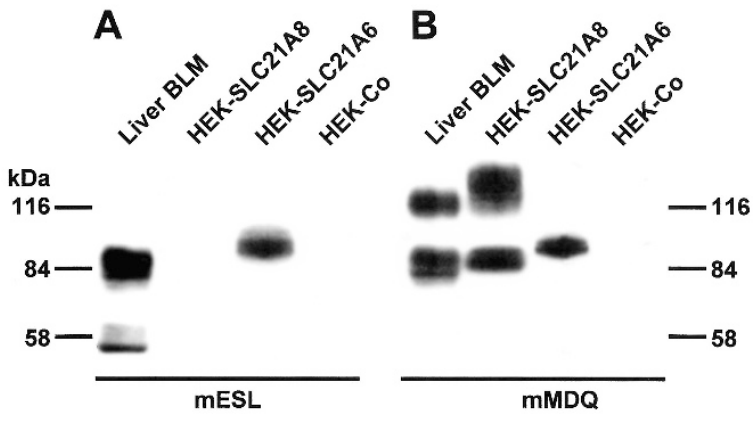

Figure 2.

Detection of human SLC21A6 and SLC21A8 by immunoblotting. Membrane preparations from human liver (liver basolateral membrane [BLM]), from SLC21A6-transfected cells (König et al, 2000a) (HEK-SLC21A6), SLC21A8transfected cells (König et al, 2000b) (HEK-SLC21A8), and control-transfected HEK293 (human embryonic kidney) cells (HEK-Co) were separated by SDSPAGE. A, The antibody mESL (at a dilution of $24 \mu \mathrm{g} / \mathrm{mL}$ in TBS-T) detected only SLC21A6 in these samples. The weak signal with a molecular mass of about $60 \mathrm{kd}$ in BLM resulted from the core-glycosylated form of SLC21A6 (König et al, 2000a). B, The antibody $\mathrm{mMDQ}$ (at a dilution of $6 \mu \mathrm{g} / \mathrm{mL}$ in TBS-T) detected both SLC21A6 and SLC21A8, resulting in two strong signals in BLM. Note that both signals detected in HEK-SLC21A8 cells resulted from SLC21A8 only, with the lower band corresponding to the core-glycosylated form of SLC21A8 (Cui et al, 2001a; König et al, 2000a).

in addition to its occurrence in a number of other tissues (Kullak-Ublick et al, 2001), was studied by means of peptide competition, using a peptide corresponding to the carboxyl terminus of SLC21A9 (LLVSGPGKKPEDSRV). Pre-adsorption with $100 \mu \mathrm{M}$ of this peptide inhibited the reactivity of $\mathrm{mESL}$ with SLC21A6 neither upon immunoblot analysis nor in immunofluorescence microscopy (data not shown).

\section{Species-Specific Reactivity of the Monoclonal Antibodies}

Cross-reactivity of both antibodies with liver samples from other species has been investigated by immunoblot analysis and immunofluorescence microscopy. As summarized in Table 1, neither of the two antibodies reacted with liver samples from dog, rat, and mouse.

\section{Immunoprecipitation of Human SLC21A6 and SLC21A8 by the Monoclonal Antibodies}

The capability of the monoclonal antibodies to precipitate SLC21A6 and SLC21A8 was studied using a basolateral membrane preparation from human liver

Table 1. Specificity and Properties of the Monoclonal Antibodies mESL and mMDQ

\begin{tabular}{|c|c|c|c|c|c|c|c|c|c|c|}
\hline \multirow[b]{3}{*}{$\mathrm{mAb}$} & \multirow[b]{3}{*}{ Antigen } & \multirow[b]{3}{*}{ Isotype } & \multicolumn{8}{|c|}{ Reactivity } \\
\hline & & & \multirow[b]{2}{*}{ Immunoblot } & \multirow[b]{2}{*}{$\mathrm{IF}^{a}$} & \multirow[b]{2}{*}{$\mathrm{HHC}^{a}$} & \multirow[b]{2}{*}{$\mid P^{a}$} & \multicolumn{4}{|c|}{ Species } \\
\hline & & & & & & & Human & Dog & Rat & Mouse \\
\hline mESL & aa671-691 & $\lg M$ & $++{ }^{b}$ & +++ & $-{ }^{b}$ & ++ & SLC21A6 & - & - & - \\
\hline $\mathrm{mMDQ}$ & aa1-23 & $\lg G$ & +++ & ++ & +++ & +++ & SLC21A6/8 ${ }^{c}$ & - & - & - \\
\hline
\end{tabular}

\footnotetext{
${ }^{a} \mathrm{IF}$, immunofluorescence microscopy; IHC, immunohistochemistry with formalin-fixed, paraffin-embedded tissues; IP, immunoprecipitation; mESL, monoclonal antibody ESL; mMDQ, monoclonal antibody MDQ.

$b+-+++$, immunoreactivity of antibodies; - , no reactivity detected in liver.

${ }^{c}$ SLC21A6/8: SLC21A6 and SLC21A8.
} 
(liver BLM) and a membrane preparation from HEKSLC21A6 cells as a sample containing only SLC21A6. To avoid interference by mouse immunoglobulins in the precipitates, SLC21A6 and SLC21A8 were detected in the precipitates by the antisera pESL and pSKT, respectively. As shown in Figure 3A, SLC21A6 was detected in the precipitates from liver BLM and HEK-SLC21A6 membranes with either mESL or $\mathrm{mMDQ}$, indicating that both monoclonal antibodies were able to precipitate SLC21A6. When the antibodies were omitted during the precipitation, no SLC21A6 could be found in the precipitates. In contrast to SLC21A6, SLC21A8 was only detected in the precipitate from liver BLM with $\mathrm{MMDQ}$ (Fig. 3B), indicating that only $\mathrm{mMDQ}$ is capable of precipitating both SLC21A6 and SLC21A8.

\section{Cellular Localization of Human SLC21A6 and SLC21A8}

The monoclonal antibodies were tested in immunofluorescence microscopy using both frozen tissue sections of human liver and HEK293 cells expressing recombinant SLC21A6 or SLC21A8. Both mESL and mMDQ stained the sinusoidal membrane in acetonefixed frozen sections of liver (red in Fig. 4). Because of the cross-reactivity of MMDQ with SLC21A6 and SLC21A8, and because of the presence of both SLC21A6 and SLC21A8 in the basolateral hepatocyte membrane, the red fluorescence in Figure 4, C and D resulted from both transporters. No overlapping staining of SLC21A6 or SLC21A8 (both red) with the apical export pump MRP2, stained with the antibody EAG5 (Büchler et al, 1996) (green), was observed, confirming the basolateral localization of SLC21A6 and SLC21A8. In contrast to liver, human brain and kidney samples did not show detectable immunoreactivity with $\mathrm{mMDQ}$ and mESL (figure not shown).

In HEK293 cells expressing recombinant SLC21A6 or SLC21A8, mMDQ detected both proteins (Fig. 5, A and $B)$, consistent with the immunoblot analysis. In contrast, mESL, which recognizes specifically SLC21A6 in immu-

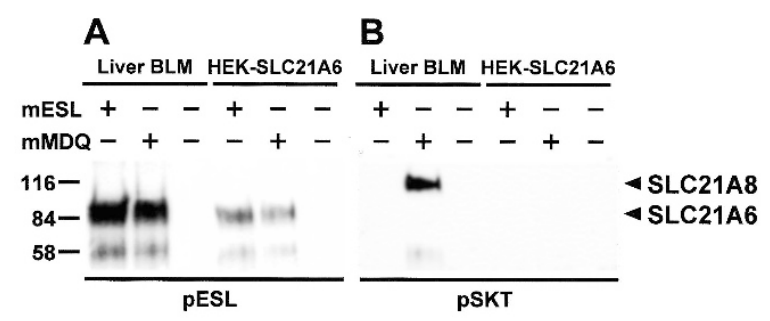

Figure 3.

Immunoprecipitation of human SLC21A6 and SLC21A8. Membrane proteins from human liver (BLM) and from SLC21A6-transfected HEK293 cells (HEKSLC21A6) were immunoprecipitated with $\mathrm{mESL}$ or $\mathrm{mMDQ}$. The precipitates were subsequently separated by SDS-PAGE. A, SLC21A6 in the precipitates was detected by the SLC21A6-specific antiserum pESL (König et al, 2000a). Both monoclonal antibodies $\mathrm{mESL}$ and $\mathrm{MMDQ}$ precipitated SLC21A6 from liver BLM and from HEK-SLC21A6 cells. SLC21A6 was not precipitated when the antibodies were omitted. B, The immunoblot shown in panel A was stripped and incubated with the SLC21A8-specific antiserum pSKT (König et al, 2000b). SLC21A8 was detected only in the precipitate from human liver membranes using the $\mathrm{mAb} \mathrm{mMDQ}$, showing that $\mathrm{mMDQ}$ also precipitated SLC21A8. noblot analysis, was also specific for SLC21A6 in immunofluorescence microscopy. Only SLC21A6 in HEKSLC21A6 cells was stained (Fig. 5C), whereas no SLC21A8 staining was observed in HEK-SLC21A8 cells (Fig. 5D). Both antibodies could also be used with formaldehyde-fixed samples (cells and frozen sections; not shown).

\section{Detection of SLC21A6 and SLC21A8 in Frozen Specimens of Hepatocellular Carcinoma and in Hepatocellular Carcinoma Cell Lines}

Membrane proteins of normal human liver and the corresponding HCC samples from three patients were studied by immunoblot analysis. For this purpose, we took advantage of the cross-reactivity of $\mathrm{mMDQ}$ with both SLC21A6 and SLC21A8 and the different molecular masses of both glycoproteins, allowing the simultaneous detection of both transporters. As shown in Figure 6, both SLC21A6 and SLC21A8 were present in normal liver samples, but the level of SLC21A8 was strongly reduced in the corresponding $\mathrm{HCC}$ samples. The level of SLC21A6 in the HCC samples appeared to be reduced, but to a lesser extent than that of SLC21A8. SLC21A6 was not detectable in the hepatocellular carcinoma cell lines HepG2 and PLC (Fig. 6). No SLC21A8 protein was observed in HepG2 cells, whereas a small amount of this protein was detectable in PLC cells (Fig. 6). The antibodies $\mathrm{mESL}$ and $\mathrm{mMDQ}$ were also studied by immunofluorescence microscopy of HCC and corresponding liver tissue (Fig. 7). Both antibodies stained the basolateral membrane of hepatocytes in the cirrhotic liver (Fig. 7, B and D). A decrease in immunofluorescent areas and an irregular staining pattern was observed in the HCC sample (Fig. 7, A and C). In HCC, the apical export pump MRP2, examined for comparison, covered the luminal membrane of pseudo-glandular structures (Fig. 7, A and C) in a pattern similar to the one described recently (Nies et al, 2001).

\section{Immunohistochemistry on Paraffin Sections: Detection of SLC21A6 and SLC21A8 in Hepatocellular Carcinoma but Not in Cholangiocarcinoma and Metastases in the Liver}

Using a microwave oven-based antigen retrieval protocol (see Materials and Methods), we obtained specific immunostaining of human liver and HCC tissue with antibody $\mathrm{mMDQ}$ (Fig. 8, A-F). The staining pattern of normal liver tissue (Fig. 8F) was similar to that observed by immunofluorescence microscopy on frozen sections. Thus, there was predominant staining of the basolateral membrane of the hepatocytes. The immunostaining was accentuated in central areas of the liver lobules, whereas in periportal areas the staining was weak or absent. Intrahepatic bile ducts were consistently negative.

A series of 12 hepatocellular carcinomas was studied as paraffin-embedded biopsy material. In 10 of these cases, positive reactions with antibody $\mathrm{mMDQ}$ were obtained (Fig. 8, A-C). The distribution of the SLC21A6/SLC21A8 immunostaining was variable and always focal and heterogeneous, ranging from a few 

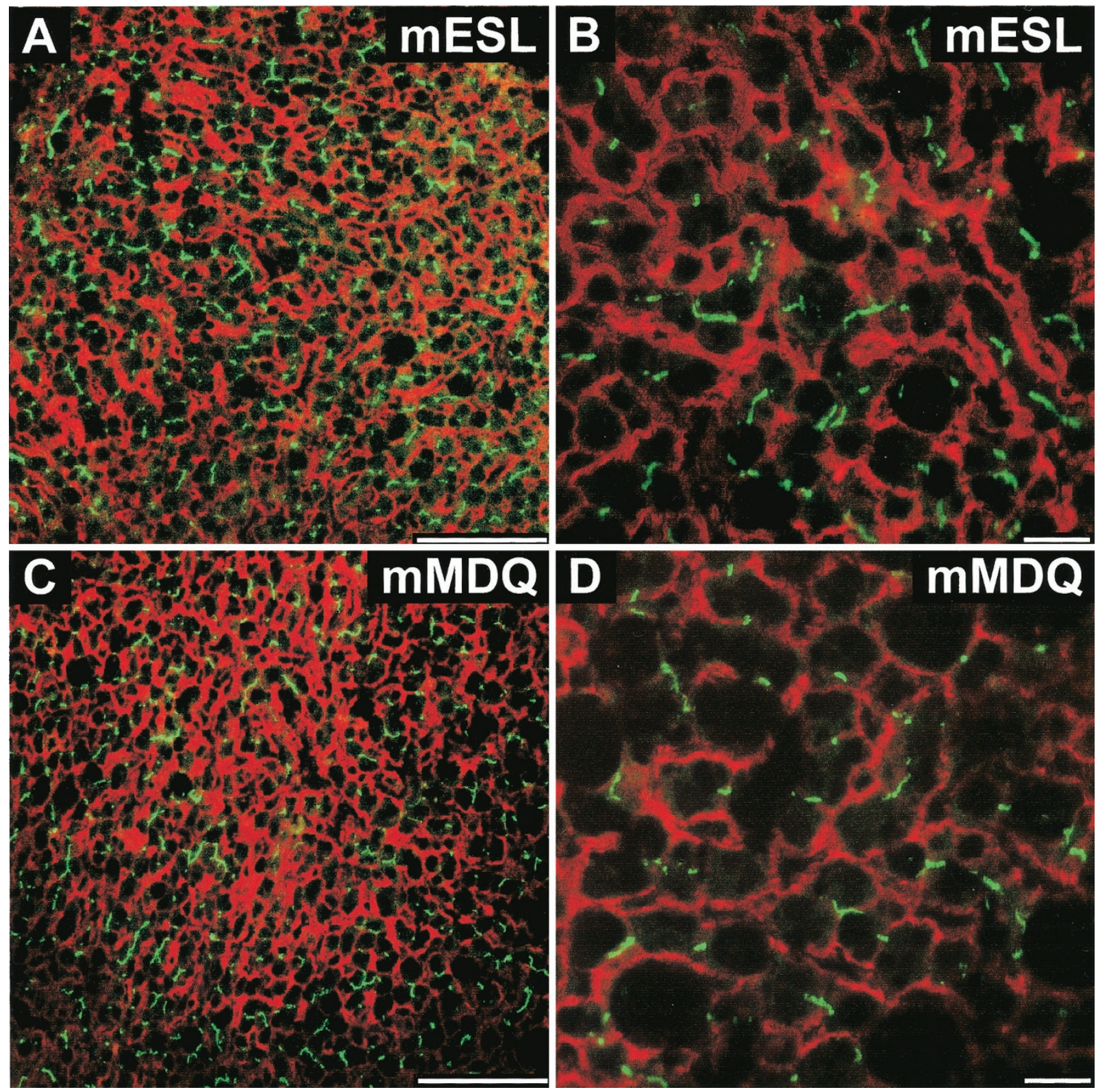

Figure 4.

Immunolocalization of SLC21A6 and SLC21A8 in human liver. Cryosections of normal human liver (5- $\mu \mathrm{m}$ thickness) were incubated either with the $\mathrm{mESL}$ antibody (red in A, B) or with the $\mathrm{mMDQ}$ antibody (red in C, D). For staining of the canalicular membrane domain, the antiserum EAG5 served to detect the apical conjugate export pump MRP2 (Büchler et al, 1996) (green in A-D). Both antibodies detected the respective SLC21A isoform in the basolateral membrane of hepatocytes. Bars in $A$ and $C, 100 \mu \mathrm{m}$; bars in $B$ and $D, 20 \mu \mathrm{m}$.

positive tumor cell groups (about $10 \%$ of the tumor cells) to more extensive tumor areas (up to about $30 \%$ of the tumor cells). The cellular staining was concentrated at the tumor cell plasma membrane and, in contrast to the staining pattern of normal hepatocytes, did not show recognizable polarity. Two cases of hepatocellular carcinomas were negative for $\mathrm{mMDQ}$ (not shown). In contrast, all 11 cases of cholangiocarcinoma of the liver were completely negative for mMDQ (Fig. 8D), including corresponding lymph node metastases in the hepatoduodenal ligament studied in two cases. The same was true for all liver metastases derived from adenocarcinomas of the colon, rectum, and pancreas, which all lacked any immunostaining of the tumor cells (Fig. 8E). Surrounding liver tissue revealed positive hepatocellular staining for $\mathrm{mMDQ}$ (Fig. 8, D and E).

In some, but not all, specimens, we observed a certain cytoplasmic cross-reactivity with mesenchymal stromal cells including tumor stroma myofibroblasts and particularly endothelial cells of blood vessels in portal fields and in the stroma of HCCs, cholangiocarcinomas, and metastases in liver (not shown). The endothelial cross-reactivity was variable and, as compared with the plasma membrane reactivity of hepatocytes, mostly of lower intensity. These 


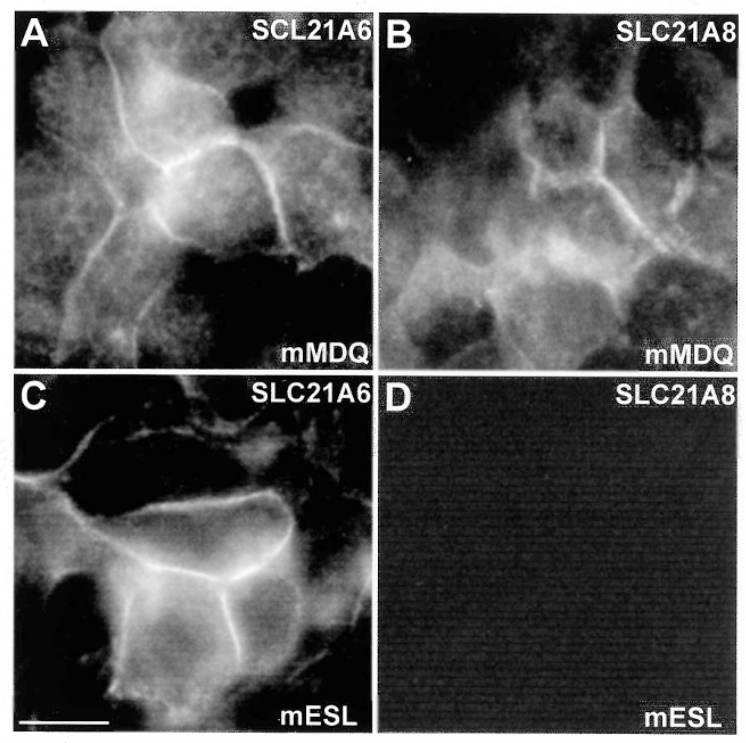

Figure 5.

Detection of human SLC21A6 and SLC21A8 in transfected HEK293 cells. SLC21A6-transfected (König et al, 2000a) and SLC21A8-transfected HEK293 cells (König et al, 2000b) were grown on glass coverslips. Cells were fixed and permeabilized with methanol/acetone. A and B, Cells incubated with antibody mMDQ at a dilution of $6 \mu \mathrm{g} / \mathrm{mL}$ in PBS. Both SLC21A6 (A) and SLC21A8 (B) were detected in the plasma membrane of the stable transfectants. $C$ and $D$, Cells were incubated with the antibody $\mathrm{mESL}(3 \mu \mathrm{g} / \mathrm{mL}$ in PBS). Only SLC21A6 was detected by mESL (C), no signal was obtained in SLC21A8-transfected cells (D). Bar represents $10 \mu \mathrm{m}$, same magnification for all panels.

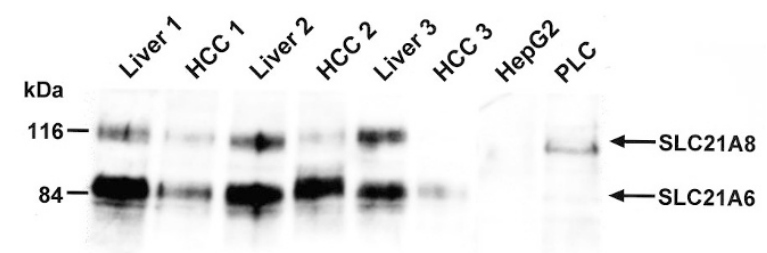

Figure 6.

Analysis of SLC21A6 and SLC21A8 in human hepatocellular carcinoma by the antibody mMDQ. Crude membranes from hepatocellular carcinoma (HCC) tissues and the surrounding normal liver of three patients and from human hepatocarcinoma HepG2 and PLC cells were separated by SDS-PAGE. SLC21A6 and SLC21A8 were detected simultaneously by the antibody mMDQ. Signals corresponding to SLC21A6 or SLC21A8 are indicated by arrows. In all three HCC samples SLC21A8 was strongly reduced and the level of SLC21A6 was diminished. SLC21A6 and SLC21A8 were not detectable in HepG2 cells. A weak signal corresponding to SLC21A8 was detected in PLC cells.

cross-reactions did not obscure the assessment of the specific SLC21A6/SLC21A8 plasma membrane staining by immunohistochemistry on paraffin sections.

\section{Discussion}

In contrast to other SLC21A family members detectable in a variety of tissues (Kullak-Ublick et al, 1995; Tamai et al, 2000), human SLC21A6 and SLC21A8 have been detected exclusively in the liver (Abe et al, 1999; Hsiang et al, 1999; König et al, 2000a, 2000b). Because of their importance for hepatobiliary elimination, including the removal of bilirubin and its glucuronosides from blood circulation (Cui et al, 2001b;
König et al, 2000a, 2000b), it is desirable to have specific monoclonal antibodies for studies of the expression and localization of SLC21A6 and SLC21A8. Therefore, we have generated the monoclonal antibodies $\mathrm{MMDQ}$ and $\mathrm{mESL}$ directed against the amino and the carboxyl terminus of SLC21A6, respectively. The applicability of both antibodies for various immunologic techniques is summarized in Table 1. Both antibodies are useful for immunoblotting, immunofluorescence microscopy, and immunoprecipitation (Table 1). However, some differences in their reactivity have also been detected. In immunoblot analyses, mMDQ performs better than $\mathrm{mESL}$ because it causes less background than mESL. Also, in immunoprecipitation experiments, $\mathrm{mMDQ}$ is more efficient than mESL. In immunofluorescence experiments, on the other hand, mESL exhibits a lower background, and thus results in clearer images than $\mathrm{MMDQ}$. Only antibody MDQ works well in immunohistochemistry on paraffin sections. Both $\mathrm{mESL}$ and $\mathrm{MMDQ}$ can be used to immunoprecipitate SLC21A6 (Fig. 3A). mMDQ also precipitates SLC21A8, consistent with its crossreactivity in immunoblot analyses (Figs. 2 and 3).

Because of the high amino acid sequence identity of $80 \%$ between SLC21A6 and SLC21A8, we examined whether the antibodies cross-react with SLC21A8. As shown by immunoblotting (Figs. 2, 3, 6), the antibody mESL was specific for SLC21A6 and showed no immunoreactivity with SLC21A8. The specificity of $\mathrm{mESL}$ was consistent with the specificity of the pESL antiserum (König et al, 2000a, 2000b), directed against the same epitope of SLC21A6. In contrast to mESL, the antibody $\mathrm{MMDQ}$ reacted with both SLC21A6 and SLC21A8 (Figs. 2-6). Because of the different extent of glycosylation and, therefore, different molecular masses of both transporters in human liver (Figs. 2, 3, $6)$, this cross-reactivity of $\mathrm{mMDQ}$ turned out to be useful because both transporters could be detected simultaneously in the same tissue sample, as exemplified in Figures 2 and 6.

Peptide competition experiments have indicated that $\mathrm{mESL}$ does not react with SLC21A9, another isoform localized in the basolateral hepatocyte membrane (Kullak-Ublick et al, 2001). Because of the low sequence identity between SLC21A6 and SLC21A9 at the amino terminus, a cross-reactivity of $\mathrm{MMDQ}$ with SLC21A9 is rather unlikely. The fact that both $\mathrm{mMDQ}$ and $\mathrm{mESL}$ have not stained human brain and kidney samples suggests that they do not react with SLC21A3 (OATP-A), which is known to be present at relatively high levels in both tissues (Kullak-Ublick et al, 1995).

With regard to the species specificity of both antibodies, we have not observed immunoreactivity in liver samples from dog, rat, or mouse. Because rat Slc21a1 (Oatp1), Slc21a5 (Oatp2), and Slc21a10 (Oatp4) are strongly expressed in rat liver (Cattori et al, 2000; Reichel et al, 1999), the lack of reactivity of the monoclonal antibodies with rat liver samples suggests that they do not cross-react with these three members of the rat Slc21a family. 

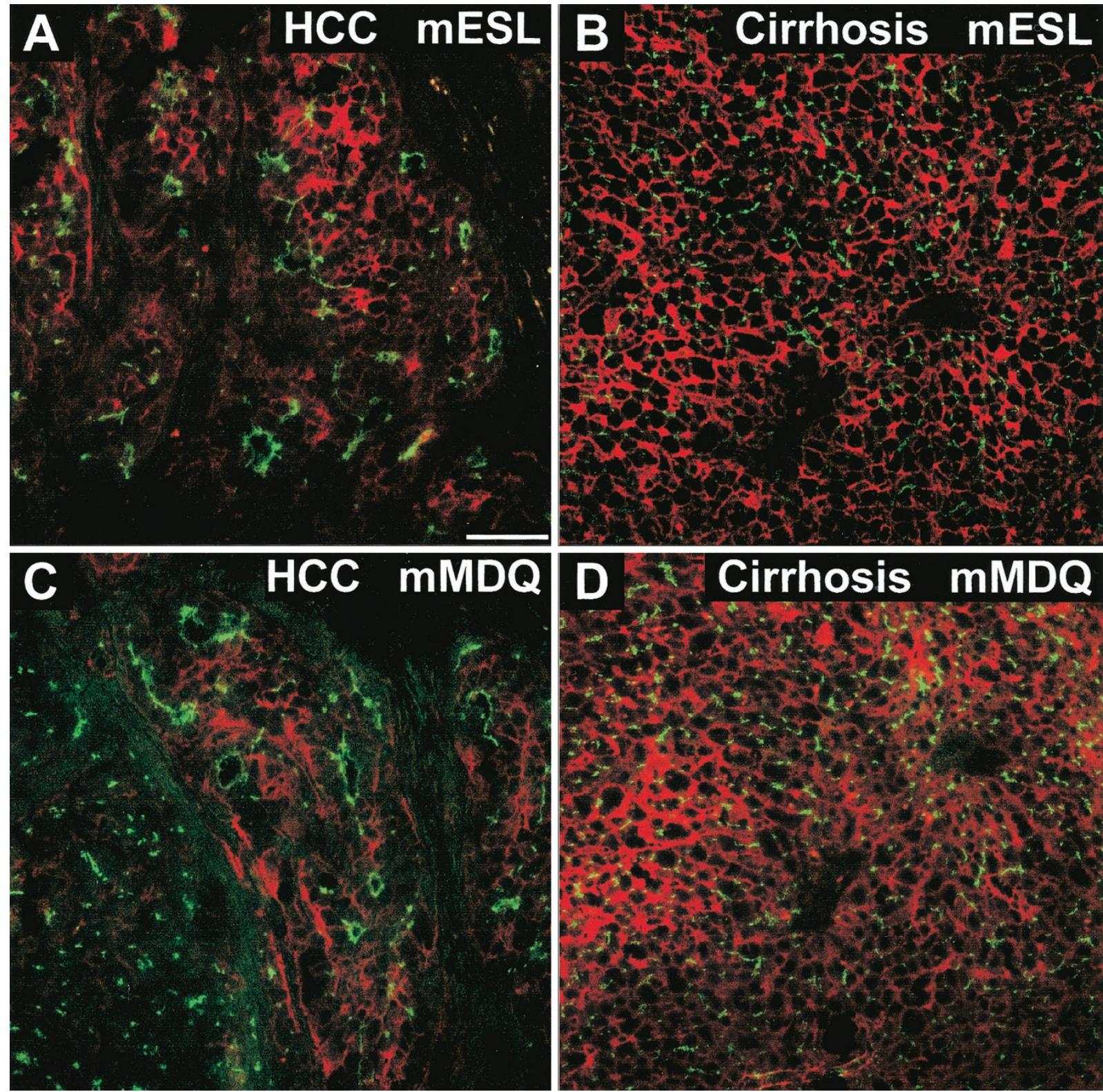

Figure 7.

Immunolocalization of SLC21A6 and SLC21A8 (both in red) in HCC (A, C) and in surrounding cirrhotic liver tissue (B, D). Canalicular domains were stained for MRP2 (green) using the EAG5 antiserum. Staining was performed as described in the legend to Figure 4, using concentrations of $\mathrm{mESL}$ and $\mathrm{mMDQ}$ of $25 \mu \mathrm{g} / \mathrm{mL}$ and 2.5 $\mu \mathrm{g} / \mathrm{mL}$, respectively. In the HCC samples, immunofluorescence staining by $\mathrm{mESL}$ and $\mathrm{mMDQ}$ was reduced as compared with the cirrhotic liver tissue and showed an irregular pattern. Bar in A, $100 \mu \mathrm{m}$; same magnification for all panels.

Our immunoblot analyses indicating that SLC21A6 and SLC21A8 proteins are not detectable in HepG2 cells (Fig. 6) are of special interest. Because SLC21A6 is a high-affinity transporter for sulfobromophthalein, with $\mathrm{a} \mathrm{K}_{\mathrm{m}}$ value of $140 \mathrm{nmol} / \mathrm{L}$ (Cui et al, 2001b), the absence of SLC21A6 from HepG2 cells is consistent with the finding that a high-affinity uptake system for sulfobromophthalein is lacking in HepG2 cells (Min et al, 1991). SLC21A6 has also not been detected in the hepatocellular carcinoma line PLC (Fig. 6). These findings suggest that some tumor cells of hepatocyte origin do not express the genes encoding these hepatocyte-specific uptake transporters involved in hepatobiliary elimination. In addition, we have ob- served down-regulation of SLC21A6 and SLC21A8 in HCC samples (Figs. 6 and 7). These observations show that the monoclonal antibodies described in this work provide useful tools for studies on the synthesis and localization of the major hepatocyte-specific uptake transporters for organic anions, SLC21A6 and SLC21A8, under physiologic and pathophysiologic conditions.

Since it has been shown that SLC21A6 and SLC21A8 are specifically expressed in hepatocytes (König et al, 2000a, 2000b), we raised the question whether these proteins, despite their partial downregulation in HCCs, might be suitable as histologic markers for hepatocellular differentiation in tumor di- 


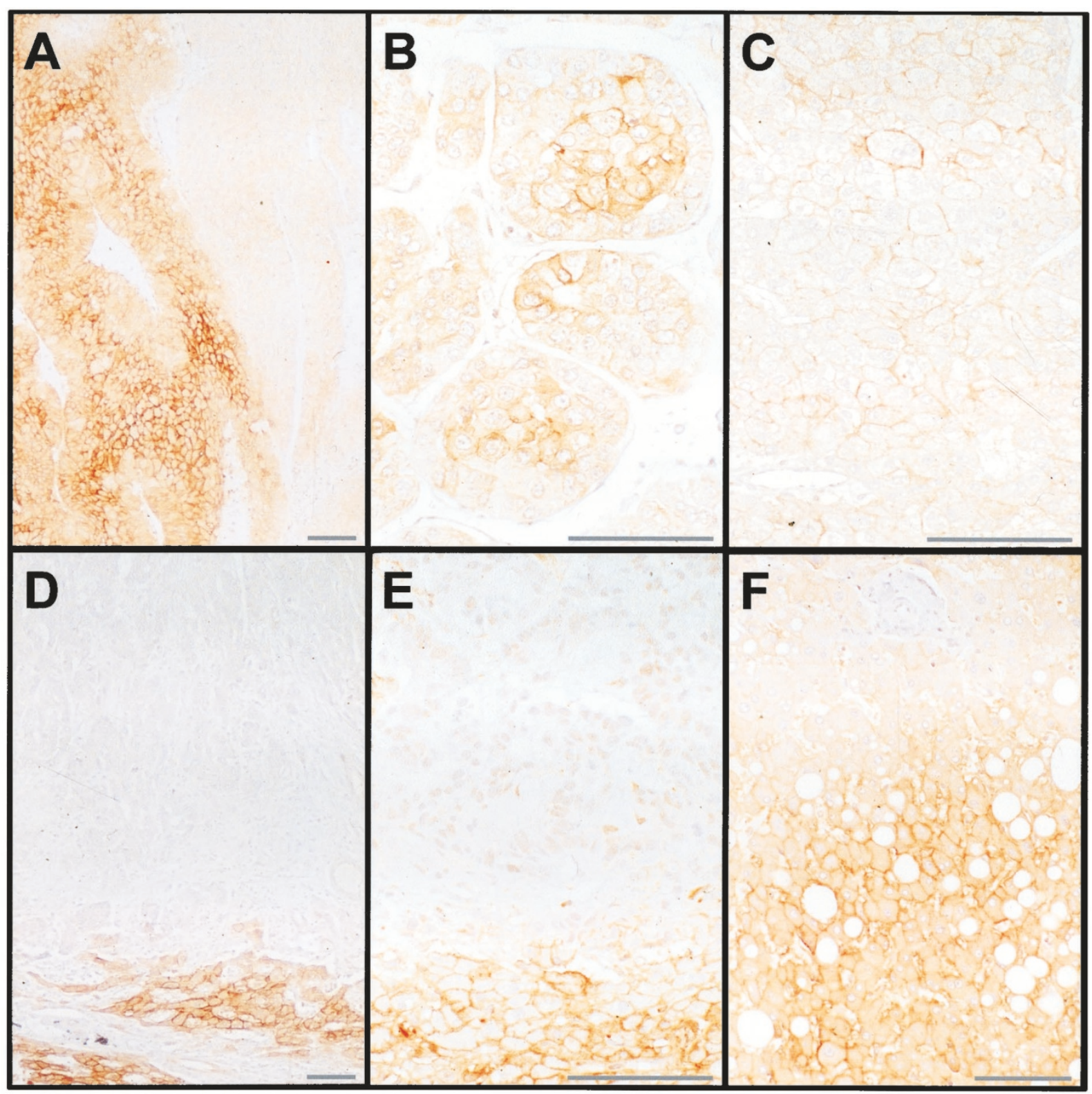

\section{Figure 8.}

Immunohistochemical localization of SLC21A6 together with SLC21A8 in paraffin sections of human liver and liver tumors (antibody mMDQ; ABC-peroxidase method). A to C, HCC specimens of three cases, showing focal SLC21A6/SLC21A8 staining with different appearance. A, Extensively positive tumor area sharply contrasting with completely negative tumor area. B, C, Heterogeneously distributed positive tumor cells. Note variable, partial, or continuous cell membrane staining of neoplastic hepatocytes. D, Cholangiocarcinoma of the liver negative for SLC21A6/SLC21A8. E, Liver metastasis of an adenocarcinoma of the colon negative for SLC21A6/SLC21A8. In D and E, note positive hepatocytes of adjacent liver tissue. F, Liver (with some fatty change) showing basolateral membrane staining of hepatocytes, preferentially in centrilobular areas (bottom part). Note negative portal field structures (top part). Bars correspond to $100 \mu \mathrm{m}$.

agnosis. The morphologic differential diagnosis of liver tumors, in particular the distinction between HCCs, cholangiocarcinomas, and liver metastases of extrahepatic adenocarcinomas may be difficult in some cases. Therefore, many attempts have been made to establish specific and distinctive immunohistochemic markers (Leong et al, 1998). Certain markers, such as carcinoembryonic antigen and cytokeratin (CK) 19 (Maeda et al, 1996), may be used to identify - with more or less specificity-the group of cholangiocarcinomas and adenocarcinoma metastases but would yield only negative evidence in the case of HCCs. Available markers for the positive identification of HCCs are limited. The most traditional marker for HCCs is $\alpha$-fetoprotein which, however, suffers from relatively low sensitivity $(<50 \%$; Chedid et al, 1990) and, in addition, is not entirely specific because it may be occasionally expressed in nonhepatocellular carcinomas such as from the stomach, ovary, and pancreas (Leong et al, 1998; Ma et al, 1993; Taketa, 1990). More recently, Hep Par 1 has been introduced as a fairly hepatocyte-specific marker (Wennerberg et al, 1993). In comparison to $\alpha$-fetoprotein, Hep Par 1 is much more sensitive (sensitivity $>80 \%$; Leong et al, 1998, Minervini et al, 1997) but again is not entirely 
specific for HCCs because certain nonhepatocellular carcinomas such as cholangiocarcinomas and stomach carcinomas may exhibit staining for Hep Par 1 (Leong et al, 1998; Minervini et al, 1997; Wennerberg et al, 1993).

The present, still preliminary data with paraffin material of human tumors suggest that SLC21A6 and SLC21A8 may complement the panel of hepatocytespecific tumor markers. We established the reactivity of $\mathrm{mAb} m \mathrm{mDQ}$ with routine paraffin-embedded human tissues. With such material, some cross-reactivity with certain mesenchymal cells, such as endothelium, was noted in some samples, but this did not hamper the diagnostic assessment of epithelial tumor cells with clear-cut cell membrane staining being the easily recognizable diagnostic criterion. In our series, we found a case-related sensitivity for HCCs of $83 \%$, clearly exceeding that of $\alpha$-fetoprotein. However, the focal nature of the SLC21A6 and SLC21A8 staining, corresponding to the down-regulation of these transporters on the biochemical level, may limit their usefulness in small needle biopsies. On the other hand, at least the present data did not reveal any staining of epithelial and carcinoma cells other than hepatocytes and HCCs, suggesting that the specificity of SLC21A6 and SLC21A8 for hepatocellular carcinoma may be very high. This needs to be confirmed by larger systematic analyses. At present, we cannot confirm recent data concerning the detection of SLC21A8 in colon carcinomas (Abe et al, 1999) because our seven cases of colorectal carcinoma metastases were completely negative for SLC21A6 and SLC21A8. It is generally accepted that in the immunohistochemical differential diagnosis of liver tumors, not a single marker, but rather selected panels of markers, yield the highest diagnostic accuracy. It now seems possible that SLC21A6 and SLC21A8 may become part of an optimized marker panel suitable for the sensitive and specific identification of HCCs in diagnostically problematic cases.

\section{Materials and Methods}

\section{Cell Culture}

HEK293 cells permanently expressing recombinant human SLC21A6 (HEK-SLC21A6) or SLC21A8 cDNA (HEK-SLC21A8), or control vector (HEK-Co) were generated and cultured as described (Cui et al, 2001b; König et al, 2000a, 2000b) in minimum essential medium. The hepatocarcinoma cell line HepG2 described before (ATCC HB-8065; Jedlitschky et al, 1997) and the primary liver carcinoma cell line PLC (ECACC No. 85061113; Leube et al, 1994) were cultured in RPMI1640 medium and DMEM, respectively. All culture media were supplemented with $10 \%$ fetal bovine serum, $100 \mu \mathrm{g} / \mathrm{mL}$ penicillin, and $100 \mathrm{U} / \mathrm{mL}$ streptomycin.

\section{Antibodies}

The antisera pESL (König et al, 2000a) and pSKT (König et al, 2000b) are directed against the carboxyl terminus of human SLC21A6 and human SLC21A8, respectively. Monoclonal mouse antibodies were generated by immunization of 9-week-old female Balb/C mice with keyhole limpet hemocyanin (KLH)-coupled peptides (Peptide Specialty Laboratories, Heidelberg, Germany). The peptide ESLNKNKHFVPSAGADSETHC, which had been used to produce the antiserum $\mathrm{pESL}$, was also used here for the generation of a mAb against the carboxyl terminus of SLC21A6. To generate a $\mathrm{mAb}$ directed against the amino terminus of SLC21A6, the peptide MDQNQHLNKTAEAQPSENKKTRYC, corresponding to the first 24 amino acid residues of SLC21A6, was used to immunize mice (Mertens et al, 1996; Schäfer et al, 1996). For each subcutaneous injection, $100 \mu \mathrm{g}$ of KLH-coupled peptide were dissolved in PBS $(137 \mathrm{mmol} / \mathrm{L} \mathrm{NaCl}, 2.7$ $\mathrm{mmol} / \mathrm{L} \mathrm{KCl}, 8 \mathrm{mmol} / \mathrm{L} \mathrm{Na}_{2} \mathrm{HPO}_{4}, 1.5 \mathrm{mmol} / \mathrm{L} \mathrm{KH}_{2} \mathrm{PO}_{4}$ ) and emulsified with an equal volume of complete Freund's adjuvant for the initial injection and with incomplete adjuvant for the booster on Day 21. The final booster injection was given without adjuvant intraperitoneally on Day 84. On Day 88, the mice were killed and spleen cells were fused with cells of a mouse myeloma cell line (P3X63-Ag8.653) at a ratio of 2:1 in the presence of polyethylene glycol (PEG 1500; Roche Molecular Biochemicals). After fusion, hybridoma cells were plated onto $8 \times 12$-well plates and cultured in hypoxanthine-aminopterin thymidine (HAT)-containing RPMI1640 medium (Life Technologies, Gaithersburg, Maryland), supplemented with $10 \%$ FCS. The immunoreactivity of hybridoma cell supernatants was screened by immunoblot analysis using membrane fractions prepared from HEKSLC21A6 cells and with immunofluorescence microscopy using HEK-SLC21A6 cells grown on glass coverslips. Positive clones were subcloned three times by limiting dilution. Ig subclasses were determined by the use of the mouse mAb isotyping kit IsoStrip (Roche Molecular Biochemicals). For large-scale antibody production, clonal hybridoma cells were cultured with serum-free RPMI1640 medium for four to five days. Supernatants containing antibodies were then collected.

\section{Immunoblot Analysis}

Membrane proteins were incubated with sample buffer for 30 minutes at $37^{\circ} \mathrm{C}$ and separated by SDS-PAGE (7.5\% separating gels). Primary antibodies, except $\mathrm{mESL}$, were diluted in TBS-T $(20 \mathrm{mmol} / \mathrm{L}$ Tris, $145 \mathrm{mmol} / \mathrm{L} \mathrm{NaCl}, 0.05 \%$ Tween 20, pH 7.6) containing $5 \%$ milk powder. The mAb mESL was diluted in TBS-T containing 10\% milk powder. The secondary antibody horseradish peroxidaseconjugated goat anti-rabbit IgG (BioRad, München, Germany) was used at a dilution of 1:2000 in TBS-T. The horseradish peroxidase-conjugated goat IgG recognizing both mouse IgG and IgM (Jackson Laboratories, West Grove, Pennsylvania) was used at a dilution of 1:5000 in TBS-T. 


\section{Immunoprecipitation}

Membrane proteins $(200 \mu \mathrm{g})$ from human liver or from HEK-SLC21A6 cells were solubilized with solubilization buffer (1\% lauryl maltoside, $150 \mathrm{mmol} / \mathrm{L} \mathrm{NaCl}, 10$ $\mathrm{mmol} / \mathrm{L}$ Hepes, $0.1 \mathrm{mmol} / \mathrm{L}$ phenylmethylsulfonyl fluoride, $\mathrm{pH} 7.4$ ) in a final volume of $500 \mu$ l for 30 minutes at $4^{\circ} \mathrm{C}$. Insoluble fractions were separated by centrifugation for 15 minutes at $4^{\circ} \mathrm{C}$ and $20,000 \times g$. Protein A Sepharose (Amersham-Pharmacia, Freiburg, Germany) was prepared for immunoprecipitation according to the manufacturer's instructions. A complex of monoclonal antibodies with protein A Sepharose was prepared by incubating the monoclonal antibodies (3 $\mu \mathrm{g} \mathrm{mMDQ}$ or $6 \mu \mathrm{g} \mathrm{mESL)} \mathrm{with} 50 \mu \mathrm{l}$ protein $\mathrm{A}$ Sepharose and binding buffer $(150 \mathrm{mmol} / \mathrm{L} \mathrm{NaCl}, 10$ $\mathrm{mmol} / \mathrm{L}$ Hepes, $0.1 \%$ lauryl maltoside, $0.1 \mathrm{mmol} / \mathrm{L}$ phenylmethylsulfonyl fluoride, $\mathrm{pH}$ 7.4) in a final volume of $500 \mu \mathrm{l}$ for 1 hour at $4^{\circ} \mathrm{C}$. After three washes with binding buffer, the antibody-protein $A$ complex was incubated with $400 \mu$ l solubilization supernatant overnight at $4^{\circ} \mathrm{C}$ on a gyratory shaker. Subsequently, the immune complex was washed twice with binding buffer and examined by immunoblotting. In control experiments, antibodies were replaced by serum-free medium.

\section{Immunofluorescence Microscopy with Cultured Cells}

HEK293 cells were grown on glass coverslips coated with poly-D-lysine $(0.1 \mathrm{mg} / \mathrm{mL})$ and induced with 10 $\mathrm{mmol} / \mathrm{L}$ sodium butyrate for 24 hours (König et al, 2000a, 2000b). The cells were fixed and permeabilized with methanol/acetone at $-20^{\circ} \mathrm{C}$, washed three times in PBS, and incubated with primary antibodies for 45 minutes at room temperature. After another three washes in PBS, the cells were incubated with Cy3conjugated goat IgG recognizing both mouse IgG and IgM (Jackson Laboratories) for 30 minutes at room temperature. The coverslips were mounted onto slides using Moviol (Calbiochem, La Jolla, California). Fluorescence micrographs were taken with a digital video camera (Hamamatsu, Hamamatsu, Japan) on an Axiovert 5100TV microscope (Carl Zeiss, Jena, Germany) and analyzed with the OpenLab imaging software (Improvision, Coventry, United Kingdom).

\section{Immunofluorescence Microscopy with Tissue Samples}

Cryostat sections of human liver and HCC obtained perioperatively were prepared for immunofluorescence studies as described (König et al, 2000a, 2000b; Nies et al, 2001). Antibodies were diluted in PBS as follows: EAG5, directed against human multidrug resistance protein 2 (MRP2, symbol ABCC2) (Büchler et al, 1996; Jedlitschky et al, 1997), at 1:100, mESL at concentrations between 5 and $25 \mu \mathrm{g} / \mathrm{mL}$, and $\mathrm{mMDQ}$ at concentrations between 1.3 and 5 $\mu \mathrm{g} / \mathrm{mL}$, Alexa Fluor488-conjugated anti-rabbit IgG (Molecular Probes, Eugene, Oregon) and Cy3conjugated goat IgG recognizing both mouse IgG and $\operatorname{lgM}$ at 1:300. Digital images were captured as de- scribed under "Immunofluorescence Microscopy with Cultured Cells."

\section{Immunohistochemistry with Paraffin-Embedded Tissues}

Paraffin blocks containing various embedded human tissues were taken from the files of the Institute of Pathology of the Philipp University of Marburg. Most of them were derived from surgical specimens that had been routinely formalin-fixed immediately after removal; some specimens were needle biopsies. The tissues included 12 cases of primary hepatocellular carcinoma (most of them moderately differentiated; no special types), 11 cases of primary cholangiocarcinoma of the liver ( 2 of them with lymph node metastases), and 12 cases of liver metastases of adenocarcinomas that originated in the colon/rectum $(n=7 ; 4$ of them mucinous) and the pancreas $(n=5)$. In addition, normal liver tissue (ie, areas in the surgical liver specimens distant from the tumors) was also studied.

For immunohistochemistry, we first established the reactivity of the monoclonal antibodies against SLC21A6/SLC21A8 with paraffin sections of formalinfixed tissue. While antibody mESL did not produce specific immunoreactions on human liver tissue, we succeeded in establishing an effective protocol for antibody $\mathrm{mMDQ}$ using a particular antigen retrieval step as follows: $3-$ to $4-\mu \mathrm{m}$ thick paraffin sections were mounted on SuperFrost Plus glass slides (Menzel, Braunschweig, Germany), incubated at $60^{\circ} \mathrm{C}$, deparaffinized with xylene, and then incubated in absolute ethanol. Endogenous peroxidase activity was blocked by incubation with $1 \% \mathrm{H}_{2} \mathrm{O}_{2}$ in methanol for 30 minutes. After rinsing in distilled water, sections were treated by microwave oven heating in a plastic

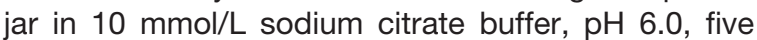
times for 5 minutes at $600 \mathrm{~W}$ (Shi et al, 1991). After cooling to room temperature, endogenous biotin was blocked using avidin-biotin-blocking kit (Vector, Burlingame, California). After incubation with 10\% normal horse serum in PBS, primary antibody $\mathrm{mMDQ}$ was applied to the sections at a dilution of $1: 100$ of the original concentration of $125 \mu \mathrm{g} / \mathrm{mL}$ overnight at $4^{\circ} \mathrm{C}$, using special coverplates (Sequenza system; Shandon, Frankfurt/Main, Germany). Bound antibodies were detected using biotinylated horse anti-mouse IgG (Vector) diluted 1:100 in PBS (30 minutes, room temperature). This was followed by the avidin-biotinperoxidase complex (ABC) method (Vectastain Elite $A B C-K i t ;$ Vector). The staining reaction was developed using 3,3'-diaminobenzidine and $\mathrm{H} 2 \mathrm{O} 2$; for mild counterstaining, Mayer's hematoxylin solution was used. For negative controls, the primary antibody was replaced by buffer or an irrelevant mAb. Photomicrographs were taken using an Olympus $\mathrm{AH} 3$ photomicroscope and Kodak EPY 64T color film.

\section{Acknowledgements}

We thank Dr. W. J. Hofmann, Institute of Pathology, Heidelberg University, for kindly providing deep- 
frozen human liver and hepatocellular carcinoma samples, Dr. G. Jedlitschky, Heidelberg, for providing membrane preparations from mouse liver, $\mathrm{H}$. Schumacher, Heidelberg, for help during cell culture work, and Viktorya Morokina and Kerstin Aschenbroich, Marburg, for their help with the immunohistochemical study of paraffin-embedded specimens.

\section{References}

Abe T, Kakyo M, Tokui T, Nakagomi R, Nishio T, Nakai D, Nomura H, Unno M, Suzuki M, Naitoh T, Matsuno S, and Yawo $\mathrm{H}$ (1999). Identification of a novel gene family encoding human liver-specific organic anion transporter LST-1. J Biol Chem 274:17159-17163.

Büchler M, König J, Brom M, Kartenbeck J, Spring H, Horie T, and Keppler D (1996). cDNA cloning of the hepatocyte canalicular isoform of the multidrug resistance protein, cMrp, reveals a novel conjugate export pump deficient in hyperbilirubinemic mutant rats. J Biol Chem 271:15091-15098.

Cattori V, Hagenbuch B, Hagenbuch N, Stieger B, Ha R, Winterhalter KE, and Meier PJ (2000). Identification of organic anion transporting polypeptide 4 (Oatp4) as a major full-length isoform of the liver-specific transporter-1 (rlst-1) in rat liver. FEBS Lett 474:242-245.

Chedid A, Chejfec G, Eichorst M, Villamil F, Terg R, Telenta $\mathrm{M}$, and Hojman R (1990). Antigenic markers of hepatocellular carcinoma. Cancer 65:84-87.

Cui Y, König J, and Keppler D (2001a). Vectorial transport by double-transfected cells expressing the human uptake transporter SLC21A8 and the apical export pump ABCC2. Mol Pharmacol 60:934-943.

Cui Y, König J, Leier I, Buchholz U, and Keppler D (2001b). Hepatic uptake of bilirubin and its conjugates by the human organic anion transporter SLC21A6. J Biol Chem 276:96269630.

Hsiang B, Zhu Y, Wang Z, Wu Y, Sasseville V, Yang WP, and Kirchgessner TG (1999). A novel human hepatic organic anion transporting polypeptide (OATP2). Identification of a liver-specific human organic anion transporting polypeptide and identification of rat and human hydroxymethylglutarylCoA reductase inhibitor transporters. J Biol Chem 274: 37161-37168.

Jansen PL (2000). Foreword: from classic bile physiology to cloned transporters. Semin Liver Dis 20:245-250.

Jedlitschky G, Leier I, Buchholz U, Hummel-Eisenbeiss J, Burchell B, and Keppler D (1997). ATP-dependent transport of bilirubin glucuronides by the multidrug resistance protein MRP1 and its hepatocyte canalicular isoform MRP2. Biochem J 327:305-310.

Keppler D and Arias IM (1997). Introduction: Transport across the hepatocyte canalicular membrane. FASEB J 11:15-18.

König J, Cui Y, Nies AT, and Keppler D (2000a). A novel human organic anion transporting polypeptide localized to the basolateral hepatocyte membrane. Am J Physiol Gastrointest Liver Physiol 278:G156-G164.

König J, Cui Y, Nies AT, and Keppler D (2000b). Localization and genomic organization of a new hepatocellular organic anion transporting polypeptide. J Biol Chem 275:2316123168.
Kullak-Ublick GA, Hagenbuch B, Stieger B, Schteingart CD, Hofmann AF, Wolkoff AW, and Meier PJ (1995). Molecular and functional characterization of an organic anion transporting polypeptide cloned from human liver. Gastroenterology 109:1274-1282.

Kullak-Ublick GA, Ismair MG, Stieger B, Landmann L, Huber R, Pizzagalli F, Fattinger K, Meier PJ, and Hagenbuch B (2001). Organic anion-transporting polypeptide B (OATP-B) and its functional comparison with three other OATPs of human liver. Gastroenterology 120:525-533.

Kullak-Ublick GA, Stieger B, Hagenbuch B, and Meier PJ (2000). Hepatic transport of bile salts. Semin Liver Dis 20: 273-292.

Leong AS-Y, Sormunen RT, Tsui WMS, and Liew CT (1998). Hep Par 1 and selected antibodies in the immunohistological distinction of hepatocellular carcinoma from cholangiocarcinoma, combined tumours and metastatic carcinoma. Histopathology 33:318-324.

Leube RE, Leimer U, Grund C, Franke WW, Harth N, and Wiedenmann B (1994). Sorting of synaptophysin into special vesicles in nonneuroendocrine epithelial cells. J Cell Biol 127:1589-1601.

Ma CK, Zarbo RJ, Frierson HF Jr, and Lee MW (1993). Comparative immunohistochemical study of primary and metastatic carcinomas of the liver. Am J Clin Pathol 99:551557.

Maeda T, Kajiyama K, Adachi E, Takenaka K, Sugimachi K, and Tsuneyoshi M (1996). The expression of cytokeratins 7, 19 , and 20 in primary and metastatic carcinomas of the liver. Mod Pathol 9:901-909.

Mertens C, Kuhn C, and Franke WW (1996). Plakophilins 2a and $2 \mathrm{~b}$ : Constitutive proteins of dual location in the karyoplasm and the desmosomal plaque. J Cell Biol 135:10091025.

Min AD, Goeser T, Liu R, Campbell CG, Novikoff PM, and Wolkoff AW (1991). Organic anion transport in HepG2 cells: Absence of the high-affinity, chloride-dependent transporter. Hepatology 14:1217-1223.

Minervini MI, Demetris AJ, Lee RG, Carr BI, Madariaga J, and Nalesnik MA (1997). Utilization of hepatocyte-specific antibody in the immunocytochemical evaluation of liver tumors. Mod Pathol 10:686-692.

Nies AT, König J, Pfannschmidt M, Klar E, Hofmann WJ, and Keppler D (2001). Expression of the multidrug resistance proteins MRP2 and MRP3 in human hepatocellular carcinoma. Int J Cancer 94:492-499.

Reichel C, Gao B, Van Montfoort J, Cattori V, Rahner C, Hagenbuch B, Stieger B, Kamisako T, and Meier PJ (1999). Localization and function of the organic anion-transporting polypeptide Slc21a6 in rat liver. Gastroenterology 117:688695.

Schäfer S, Stumpp S, and Franke WW (1996). Immunological identification and characterization of the desmosomal cadherin Dsg2 in coupled and uncoupled epithelial cells and in human tissues. Differentiation 60:99-108.

Scharschmidt BF, Waggoner JG, and Berk PD (1975). Hepatic organic anion uptake in the rat. J Clin Invest 56:1280 1292.

Shi S-R, Key ME, and Kalra KL (1991). Antigen retrieval in formalin-fixed, paraffin-embedded tissues: An enhancement method for immunohistochemical staining based on micro- 
wave oven heating of tissue sections. J Histochem Cytochem 39:741- 748 .

Suzuki H and Sugiyama Y (2000). Transport of drugs across the hepatic sinusoidal membrane: Sinusoidal drug influx and efflux in the liver. Semin Liver Dis 20:251-263.

Taketa K (1990). Alpha-fetoprotein: Reevaluation in hepatology. Hepatology 12:1420-1432.

Tamai I, Nezu J, Uchino H, Sai Y, Oku A, Shimane M, and Tsuji A (2000). Molecular identification and characterization of novel members of the human organic anion transporter (OATP) family. Biochem Biophys Res Commun 273:251-260.
Wennerberg AE, Nalesnik MA, and Coleman WB (1993). Hepatocyte paraffin 1: A monoclonal antibody that reacts with hepatocytes and can be used for differential diagnosis of hepatic tumors. Am J Pathol 143:1050-1054. 